\title{
Sit to Stand Transfer Assisting by an Intelligent Walking-Aid
}

\author{
P. Médéric, V. Pasqui, F. Plumet and P. Bidaud \\ Laboratoire de Robotique de Paris (LRP) \\ CNRS FRE 2507 - Université Pierre et Marie Curie, Paris 6 \\ 18, route du Panorama, BP 61, 92265 Fontenay-aux-Roses, France \\ \{mederic,pasqui,plumet,bidaud\}@robot.jussieu.fr
}

\begin{abstract}
The sit to transfer assisting for elderly patients using an intelligent walkingaid is presented. This assistive device provides physical support to aid elderly with physical impairment during both the walk and the sit-to-stand transfer. In this paper, we will focus on the sit-to-stand transfer function and more precisely on the trajectory generation of the assistive device handles using interpolating cubic splines.
\end{abstract}

\section{Introduction}

Instability and falling are among the most serious problems associated with aging. Age-related changes in the neural, sensory and musculoskeletal systems can lead to balance impairments that have a tremendous impact on the ability to move safely, and the consequences of instability and falling, in terms of health care costs and quality of life, are more than significant.

A growing interest in developing intelligent assistive devices for the elderly can be noted in the past few years. Robotics technologies have been investigated to provide physical support of patients and to promote safe mobility ([1], [2], [3] [4]). The Care-O-Bot ([5], [6]) and the Nursebot [7] are personal service robots projects mainly focused on the man-machine interaction. A powerassistance device has been developed by the Ritsumeikan University [8] to provide physical support during the walk and the sit to stand transfer. This system is fixed and must be installed in a given hospital room.

The paper is organized as follow: we first present the assistive device developed in our laboratory. This assistive device provide support during the walk and also during the sit to stand transfer and is primarly intended for elderly patients. Then, the synthesis of the handles trajectory for the sit to stand and 
stand to sit transfer is presented. This synthesis is done using temporal cubic splines and is based on an analysis of experimental sit to stand transfer curves recorded on a set of elderly patients.

\section{Assistive Device}

Functional decline due to aging and fall, with its functional and psychological consequences, are responsible for the most common walking troubles associated with aging. Injuries with bone fractures and fear of fall ("post-fall" syndrome) are the main pathologies appearing after a fall. After a bone fracture on the lower limb, the medical staff must thus spent a lot of time and energy helping patients to stand up and to walk by their own self with intensive rehabilitation exercise. The "post-fall" syndrome is one of the psychological consequences of the fall. This syndrome leads to a disturbance of posture: the retropulsion. During the walk, the torso is lined backward with shuffling gait and the risk of fall increases. The elderly must also be assisted in the sit to stand transfer since patient sited-down in a retropulsion configuration cannot use properly his body to get into an antepulsion posture to provide propulsion in the direction of the motion.

Active devices for postural compensation can then free medical staff for other tasks, and help elderly people to do rehabilitation exercises with various difficulty levels. Based on this analysis of the most common walking trouble associated with aging, we design the overall kinematic of our assistive device. This assistive device has two main functions:

- Postural stability, to provide support and avoid fall during the walk.

- Verticalisation, to help elderly during stand-up and sit-down transitions.

The designed robotic system is basically a two degrees of freedom mechanism mounted on an active mobile plateform (see Fig 1).

For the sit to stand transfer, the handles must first pull slowly the patient
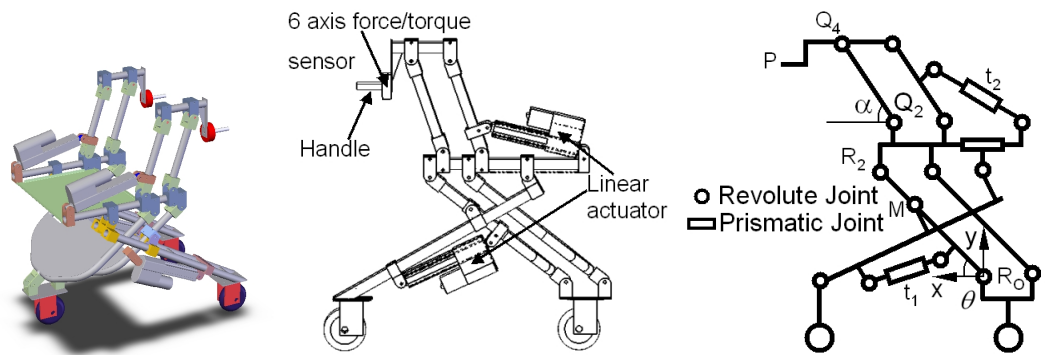

Fig. 1. Description of the assisting device

to an antepulsion configuration. Then, the handles go from this down posi- 
tion to the up position, which is the position used for walking. Obviously, the handles must remain horizontal during the whole transition. This is obtained using two parallel and independent mechanisms combined in a serial way. Details on the design of this assistive device can be found in [9].

To assist the user during the transfer the handles of the assisting device must be controlled, this is made by using the inverse kinematic model of our structure. The inverse kinematic model define the position of the tip of the handles $\mathbf{x}_{\mathbf{P}}$ in the frame $R_{0}$ for a given configuration of the linear actuator $\left(t_{1}\right.$ and $t_{2}$ ). We get this model by considering the device as a planar $2 \mathrm{R}$ (see Fig 1 ) with $\phi=\theta-\alpha$. The position of the point $\mathrm{P}$ is given by:

$$
\mathbf{x}_{\mathbf{P}}=\left(\begin{array}{c}
X_{P} \\
Y_{P}
\end{array}\right)=\left(\begin{array}{l}
O R_{2} \cos \theta-R_{2} Q_{2 x}+Q_{2} Q_{4} \cos \phi+Q_{4} P_{x} \\
O R_{2} \sin \theta+R_{2} Q_{2 y}+Q_{2} Q_{4} \sin \phi-Q_{4} P_{y}
\end{array}\right)
$$

We first get the value of $\phi$ for a given position $\mathbf{x}_{\mathbf{P}}$ of the handle, in eq. (1) we group the constant terms in a vector $\mathbf{x}_{\mathbf{P}}^{\prime}$ :

$$
\mathbf{x}_{\mathbf{P}}^{\prime}=\left(\begin{array}{c}
X_{P}^{\prime} \\
Y_{P}^{\prime}
\end{array}\right)=\left(\begin{array}{c}
O R_{2} \cos \theta+Q_{2} Q_{4} \cos \phi \\
O R_{2} \sin \theta+Q_{2} Q_{4} \sin \phi
\end{array}\right)
$$

With $\phi \in\left[0, \frac{\pi}{2}\right]$ the relation between $\phi$ and $\mathbf{x}^{\prime} \mathbf{P}$ is given by:

$$
\phi=\arccos \left(\frac{\left(X_{P}^{\prime}\right)^{2}+\left(Y_{P}^{\prime}\right)^{2}-\left(O R_{2}^{2}+Q_{2} Q_{4}^{2}\right)}{2 O R_{2} Q_{2} Q_{4}}\right)
$$

With $\phi$ known we have the following system :

$$
\mathbf{x}_{\mathbf{P}}^{\prime}=\mathbf{A}\left(\begin{array}{c}
\cos \theta \\
\sin \theta
\end{array}\right)
$$

The matrix $\mathbf{A}$ is given by:

$$
\mathbf{A}=\left(\begin{array}{cc}
O R_{2}+Q_{2} Q_{4} \cos \phi & Q_{2} Q_{4} \sin \phi \\
Q_{2} Q_{4} \sin \phi & O R_{2}+Q_{2} Q_{4} \cos \phi
\end{array}\right)
$$

After solving Eqs. (3) and (4) we need the relation between the displacement of the linear actuator $t_{1}$ and the angular variation $\theta$ and also the relation between $t_{2}$ and $\alpha$. For the lower part (see Fig (2a)) we get the following expression:

$$
t_{1}=\sqrt{M I_{1}^{2}+M I_{2}^{2}-2 M I_{1} M I_{2} \cos \left(-\pi+\varphi_{1}+\varphi_{2}+\arcsin \left(\frac{L_{1}}{L_{2}} \sin \theta\right)\right)}
$$

For the upper part (see Fig (2b)) the relation with the angle $\alpha$ and the linear displacement $t_{2}$ is given by : 


$$
t_{2}=\sqrt{Q_{1} I_{3}^{2}+\left(Q_{1} I_{4}^{2}+2 Q_{1} I_{3} Q_{1} I_{4} \cos \left(\alpha+\beta^{\prime}\right)\right) \cos \left(\alpha^{\prime}\right)}
$$

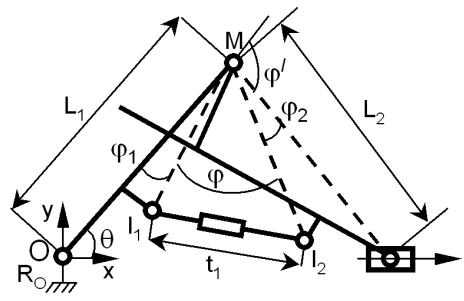

(a) Lower part

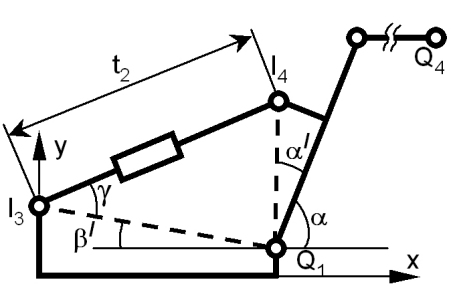

(b) Upper part

Fig. 2. Kinematic descriptions of the Lower and Upper part

In the next section, we describe the handle's trajectory synthesis needed to perform the transfer assistance.

\section{Trajectory synthesis}

In order to implement the trajectory generation (described in §3.2) for the handles of our prototype, we first do some experiments with patients and record the patient's hands trajectories and forces during assisted sit to stand and stand to sit transfers.

\subsection{Experimental trajectory analysis}

Experiments has been conducted on a set of 7 elderly patients in Charles-Foix Hospital under the supervision of gerontologists and physiotherapists.

A test platform has been developed to simultaneously record the trajectory and force interaction of elderly's hand during sit-to-stand and stand-to-sit transfer [9]. For the sit to stand transfer, the patient is seated on a chair and holds the central handle. The two caregivers hold their handles and impose the motion of the test platform according to the gerontologists and physiotherapists instructions until the patient is in a quiet standing position. The initial (down) position of the patient handle is chosen close to the knee position of the patient, forcing the patient to leave off his rather usual retropulsion configuration for an antepulsion configuration.

The reverse procedure is applied for the stand to sit transfer.

For each patient, several sit to stand and stand to sit transfer trajectories was recorded and some examples of these trajectories are given Fig 3.

Analysis of the transfer trajectories records show some interesting points: 


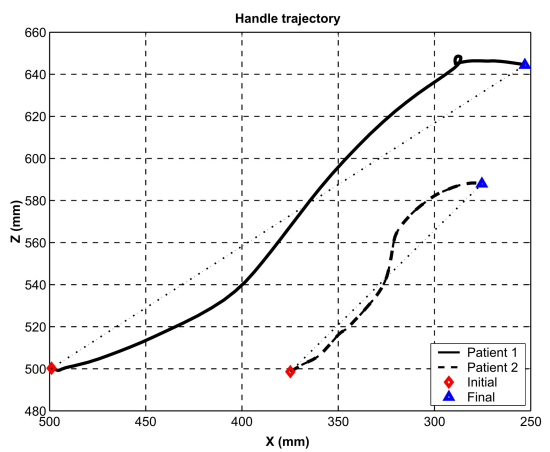

Sit to Stand transfer

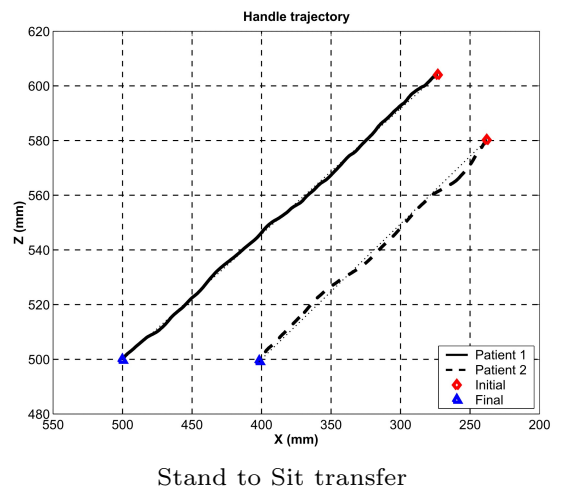

Stand to Sit transfer

Fig. 3. Trajectories for different Patient

- The sit to stand transfer trajectory follow roughly a straight line between the two end points with a normal deviation that never exceed $12 \%$ of the total trajectory length for all the patients. The sit to stand transfer trajectory is similar but with a normal deviation that do exceed $5 \%$ of the total length.

- The global shape of the trajectory is not directly related to the age, height or pathology of the patient. Moreover, the difference between the initial and final point (down and up positions of the handle) is not directly related to the height of each patient but seems to be correlated with its own personal strategy to stand up or sit down.

These items can't be considered as rules (since the number of patients is not statistically significant and since the observed trajectories are the result of an interaction between the caregivers and the patient) but rather like practical guidelines for the trajectory generation described in the next section.

\subsection{Trajectory generation}

A trajectory generation is needed to compute the desired motion of the handles of the assistive device in real time. These trajectories have no need to precisely interpolate the experimental trajectories but they rather must reproduce the global shape of the trajectories depicted in $\S 3.1$.

The trajectory generation is based on interpolating cubic splines for their interesting properties which are mainly the $\mathrm{C}^{2}$ continuity, the local deformation properties and the computational efficiency of this method (given a set of control points, interpolating cubic spline is a piecewise $3^{\text {rd }}$ order polynomial curve that interpolates these control points i.e. passes through them all).

From a functional point of view, the patient first choose the initial $\mathbf{X}_{I n i}$ and final $\mathbf{X}_{E n d}$ points of the transfer trajectory (up and down positions of the handles in the X-Z plane). The trajectory generation then automatically add 
3 other interpolating points between $\mathbf{X}_{I n i}$ and $\mathbf{X}_{E n d}$ to control the local deformation of the curve and compute the coefficients of the $3^{r d}$ order piecewise polynomial (details on the computation of these coefficients are given in the Appendix). According to the experiments done in $\S 3.1$, three points is enough to control the global shape of the transfer trajectory.

Finally, and since we use a temporal spline (i.e the $\mathbf{u}$ parameter is a linear function of time: $\mathbf{u}=\alpha_{i}$ t with $\alpha_{i}=1 / \mathrm{T}_{i}$ and $\mathrm{T}_{i}$ the time to go from the control point $\mathbf{X}_{i}$ to the control point $\mathbf{X}_{i+1}$ ), the real time computation of the trajectory begin i.e. a new point can be computed at each sampling time using a increment $\mathrm{du}=\mathrm{T}_{e} / \mathrm{T}_{i}$ of the parameter $\mathbf{u}$.

However, as mentioned early, the cubic spline is continuous up to the $2^{\text {nd }}$ derivative of $\mathbf{u}$. Recalling that $\mathbf{u}$ is a linear function of time, the trajectory is also continuous up to the $2^{\text {nd }}$ derivative of time only if $\mathbf{u}$ is the same linear function of time for the whole spline. An immediate and important consequence is that the time to go from $\mathbf{X}_{i}$ to $\mathbf{X}_{i+1}$ must be equal i.e each control points must be equally spaced in time. In other words, the choice of the added interpolating points not only control the local deformation of the curve but also the velocity profile along the trajectory.

To help elderly patient in the sit to stand (or stand to sit) transfer, we need to generate smooth trajectory both in position and time i.e. the patient's hands must be accelerated gently to attain a maximum speed and then decelerated to reach the final position. For example, to get a parabolic velocity profile for the entire spline, elementary calculation gives that the 3 additional points $\left\{X_{1}, X_{2}, X_{3}\right\}$ must be chosen such that:

$$
X_{1}-X_{\text {Ini }}=\frac{5}{32} L \quad X_{2}-X_{\text {Ini }}=\frac{L}{2} \quad X_{E n d}-X_{3}=\frac{5}{32} L
$$

with $\mathrm{L}$ the total length of the trajectory. Some examples of the interpolating spline trajectory for $5 \%$ and $10 \%$ of the total length normal deviation are given Fig. 4.

A comparison between experimental trajectories and the resulting spline trajectories for the sit to stand transfer is given Fig. 5 .

\section{Conclusion}

An assistive device for elderly patients has been developed to provide support during the walk and also during the sit to stand transfer. The synthesis of the handles trajectory for the sit to stand and stand to sit transfer has been presented. This synthesis is done using temporal cubic splines and is based on an analysis of experimental sit to stand transfer curves recorded on a set of elderly patients. This results in a automatic and versatile trajectory generator that can be adapted to the personal feeling and strategy to stand up and sit down of the patient.

Since force sensor are integrated into the handles, this device can also be a 
Sit to Stand Transfer Assisting by an Intelligent Walking-Aid
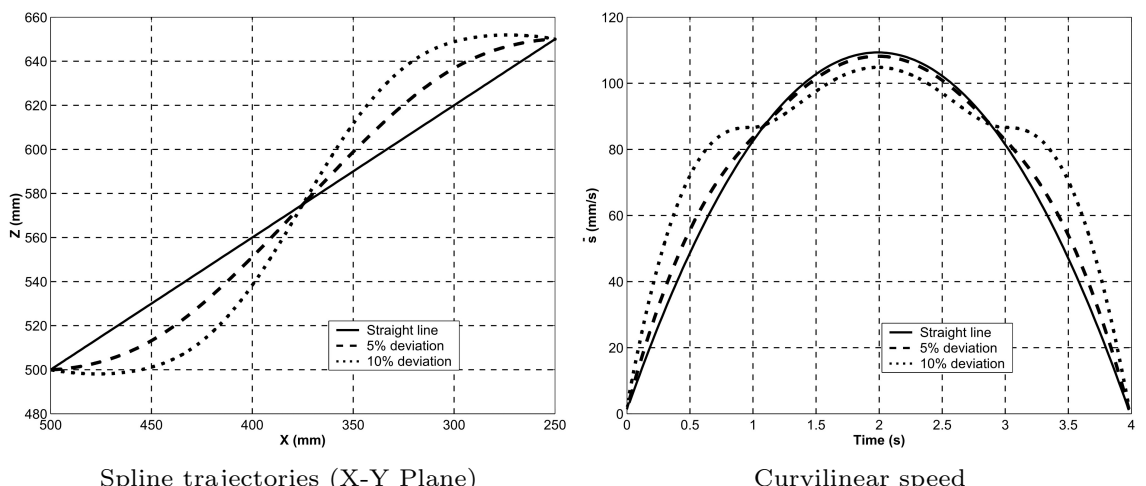

Fig. 4. Interpolating trajectories exemples
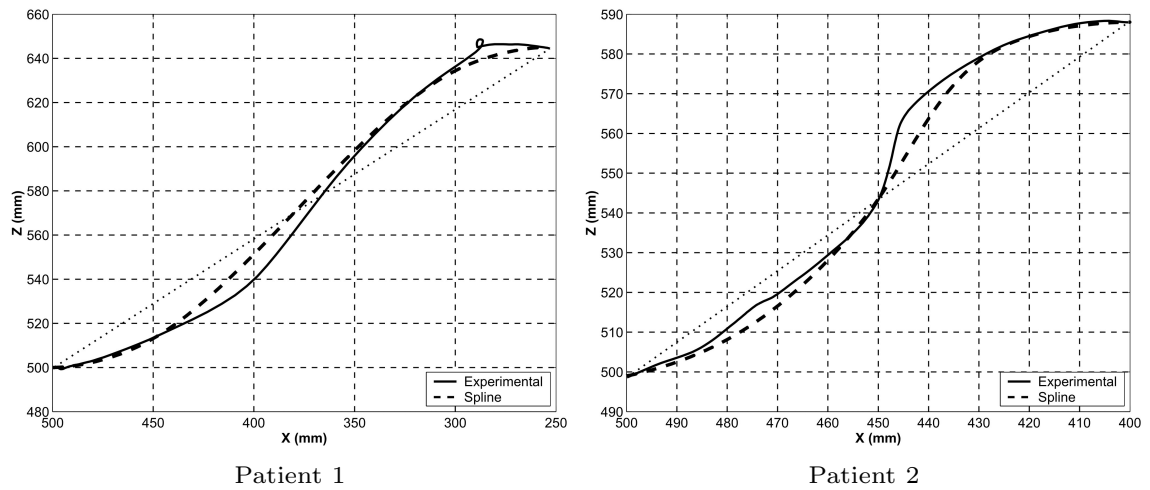

Fig. 5. Experimental and interpolating spline trajectories

useful tools for gerontologist to better understand walking troubles and to prevent falls as well as a powerful programmable device for the monitoring and rehabilitation of the lower limbs.

\section{Acknowledgments}

This work is partly supported by the French RNTS (Réseau National des Technologies de la Santé) program under grant Nº 02B0414 (Monimad Project).

\section{Appendix}

Let $\mathbf{X}=\left\{x_{o}, x_{1}, \ldots x_{n}\right\}$ be a set of $(\mathrm{n}+1)$ points equally spaced in time and $\mathrm{S}$ the interpolating cubic spline passing trough each point $x_{i}$. On each segment $\left[x_{i} x_{i+1}\right]$, the $3^{\text {rd }}$ order spline polynomial is written as:

$$
S_{i}(u)=a_{i} u^{3}+b_{i} u^{2}+c_{i} u+d_{i} \quad i=0 \ldots(n-1)
$$


with $\mathrm{u}=[0 \ldots 1]$ on each segment.

The first derivative $S_{i}^{\prime}$ and second derivative $S_{i}^{\prime \prime}$ of $S_{i}$ with respect to $u$ are given by :

$$
S_{i}^{\prime}(u)=3 a_{i} u^{2}+2 b_{i} u+c_{i} \quad \text { and } \quad S_{i}^{\prime \prime}(u)=6 a_{i} u+2 b_{i} \quad i=0 \ldots(n-1)
$$

For $(\mathrm{n})$ segments, we must then compute $(4 \mathrm{n})$ unknown coefficients. The interpolation conditions give $(n+1)$ equations and the continuity conditions on S, S' and S" give 3(n-1) equations which leads to (4n-2) equations. The two remaining equations are given by the boundary conditions of the $S$ curve i.e the initial velocity $V_{I n i}$ on the first segment and the final velocity $V_{E n d}$ on the last segment.

- The interpolation condition is: $S_{i}(u=0)=x_{i}$ which leads to:

$$
d_{i}=x_{i} \quad i=0 \ldots(n-1)
$$

- The continuity condition on $S_{i}^{\prime \prime}$ is: $S_{i}^{\prime \prime}(u=1)=S_{i+1}^{\prime \prime}(u=0)$ which leads to: $6 a_{i}+2 b_{i}=2 b_{i+1}$ and finally:

$$
a_{i}=\frac{b_{i+1}-b_{i}}{3} \quad i=0 \ldots(n-1)
$$

Note that an unknown additional parameter $b_{n}$ must be added to get a valid equation for $\mathrm{i}=\mathrm{n}-1$.

- The continuity condition on $S_{i}$ is: $S_{i}(u=1)=S_{i+1}(u=0)$ which leads to: $a_{i}+b_{i}+c_{i}+d_{i}=d_{i+1}$ and finally:

$$
c_{i}=\left(x_{i+1}-x_{i}\right)-\frac{b_{i+1}}{3}-\frac{2 b_{i}}{3} \quad i=0 \ldots(n-1)
$$

- The continuity condition on $S_{i}^{\prime}$ is: $S_{i}^{\prime}(u=1)=S_{i+1}^{\prime}(u=0)$ which leads to: $3 a_{i}+2 b_{i}+c_{i}=c_{i+1}$. Replacing $a_{i}$ and $c_{i}$ by their values given in Eq. (9) and (10), we have:

$$
b_{i}+4 b_{i+1}+4 b_{i+2}=3\left[\left(x_{i+2}-x_{i+1}\right)-\left(x_{i+1}-x_{i}\right)\right] \quad i=0 \ldots(n-2)
$$

- The two boundary conditions give two other equations.

$S_{0}^{\prime}(u=0)=V_{I n i}=c_{0}$. Replacing in Eq. (10), this gives:

$$
2 b_{0}+b_{1}=3\left[\left(x_{1}-x_{0}\right)-V_{\text {Ini }}\right]
$$

And the boundary condition on the last segment:

$S_{n-1}^{\prime}(u=1)=V_{\text {Fin }}=3 a_{n-1}+2 b_{n-1}+c_{n-1}$ gives in a similar manner:

$$
b_{n-1}+b_{n}=3\left[V_{\text {Fin }}-\left(x_{n}-x_{n-1}\right)\right]
$$


Eq. (11), (12) and (13) can be put in a matrix form:

$$
\left[\begin{array}{cccccc}
2 & 1 & 0 & 0 & \ldots & 0 \\
1 & 4 & 1 & 0 & \ldots & 0 \\
0 & 1 & 4 & 1 & \ldots & 0 \\
\vdots & & \ddots & & \vdots \\
0 & \ldots & 0 & 1 & 4 & 1 \\
0 & \ldots & 0 & 0 & 1 & 2
\end{array}\right]\left[\begin{array}{c}
b_{0} \\
b_{1} \\
b_{2} \\
\vdots \\
b_{n-1} \\
b_{n}
\end{array}\right]=\left[\begin{array}{c}
3\left(\Delta x_{1}-V_{I n i}\right) \\
3\left(\Delta x_{2}-\Delta x_{1}\right) \\
3\left(\Delta x_{3}-\Delta x_{2}\right) \\
\vdots \\
3\left(\Delta x_{n}-\Delta x_{n-1}\right) \\
3\left(V_{I n i}-\Delta x_{n}\right)
\end{array}\right]
$$

with $\Delta x_{i}=x_{i}-x_{i-1}$.

Finally, the algorithm to compute the (4n) coefficients of the spline is as follow:

- Compute $d_{i}(\mathrm{i}=0 \ldots \mathrm{n}-1)$ using Eq. (8)

- Compute $b_{i}(\mathrm{i}=0 \ldots \mathrm{n})$ by inverting Eq. (14) with LU decomposition or similar algorithm.

- Compute $a_{i}$ (i=0 ... n-1) using Eq. (9)

- Compute $c_{i}(\mathrm{i}=0 \ldots \mathrm{n}-1)$ using Eq. (10)

\section{References}

1. G. Lacey, S. Mac Namara, and K. M. Dawson-Howe. Personal adaptive mobility aid for the infirm and elderly blind. Lecture Notes in Computer Science, 1458:211$220,1998$.

2. S. Dubowsky, F. Genot, S. Godding, H. Kozono, A. Skwersky, H. Yu, and L.S. Yu. Pamm - a robotic aid to the elderly for mobility assistance and monitoring: A "helping-hand" for the elderly. In IEEE Int. Conference on Robotics and Automation, pages 570-576, 2000.

3. H. Yu, M. Spenko, and S. Dubowsky. An adaptive shared control system for an intelligent mobility aid for the elderly. Autonomous Robots, 15:53-66, 2003.

4. C. Lee, S. Oh K. Kim, and J. Lee. A system for gait rehabilitation : mobile manipulator approach. In IEEE Int. Conference on Robotics and Automation, pages 3254-3259, 2002.

5. R.D. Schraft, C. Schaeffer, and T. May. Care-o-bot ${ }^{\mathrm{TM}}$ : The concept of a system for assisting elderly or disabled persons in home environments. In 24th Annual Conf. of the IEEE Industrial Electronics Society (IECON'98), pages 2476-2481, 1998.

6. B. Graf and M. Hägele. Dependable interaction with an intelligent home care robot. In IEEE Int. Conference on Robotics and Automation, pages 21-26, 2001.

7. G.Baltus et al. Towards personal service robots for the elderly. In Workshop on Interactive Robotics and Entertainment (WIRE'00), 2000.

8. K. Nagai, I. Nakanishi, and H. Hanafusa. Assistance of self-transfer of patients using a power-assisting device. In IEEE Int. Conference on Robotics and Automation, pages 4008-4015, 2003.

9. P. Médéric, J. Lozada, V. Pasqui, F. Plumet, P. Bidaud, and J.C. Guinot. An optimized design for an intelligent walking-aid. In 6th Int. Conference on Climbing on Walking Robots (CLAWAR'03), pages 53-60, 2003. 